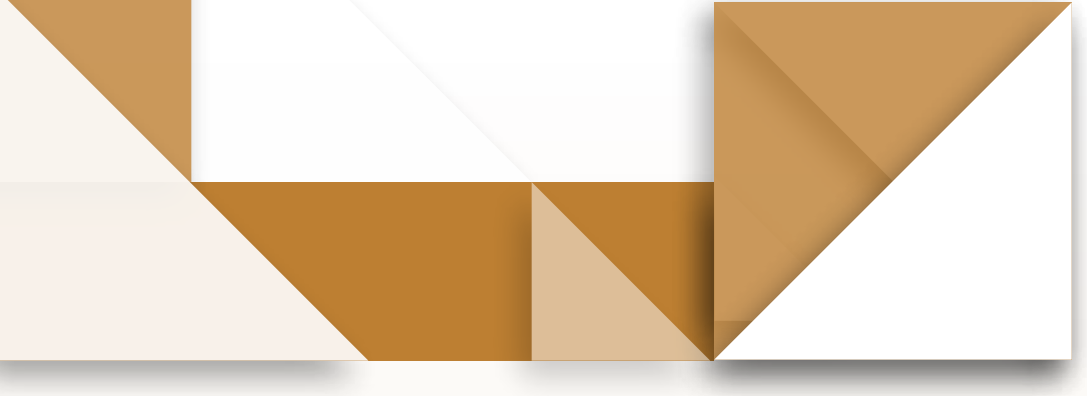

\title{
1) 25 años de la Revista TED: aportes en la consolidación del grupo de investigación IREC
}

- 25 Years of the TED Journal: Contributions in the Consolidation of the IREC Research Group

- $25^{\circ}$ aniversário da Revista TED: Contribuições para a consolidação do Grupo de Pesquisa IREC

\section{Resumen}

Con ocasión de la conmemoración de los 25 años de la revista Tecné, Episteme y Didaxis - TED, en este artículo se comunican los resultados de una investigación histórico-documental en la que, desde la perspectiva metodológica de los estudios cienciométricos, se analizan los aportes de este vehículo de circulación del conocimiento científico, en la consolidación del Grupo de Investigación Representaciones y Conceptos Científicos, IREC. El trabajo efectuado se centra tanto en el rastreo de las contribuciones emprendidas por este colectivo académico durante la vigencia de la revista - caracterizando las principales líneas de investigación, áreas temáticas, metodologías, entre otros aspectos-, como en el análisis de citas que la comunidad de especialistas ha realizado sobre algunos de estos artículos. Se concluye que, cumplido su cuarto de siglo de edad, esta revista representa un escenario fundamental en el desarrollo y dinámica de colectivos académicos como el aquí reportado, a nivel institucional, local, nacional e iberoamericano.

Palabras clave

Revista TED; Grupo IREC; didáctica de las ciencias experimentales; investigación documental; cienciometría

\section{Abstract}

On the occasion of the commemoration of the 25 years of the journal Tecné, Episteme and Didaxis - TED, this article communicates the results of a historical-documentary investigation in which, from the methodological perspective of the scientometric studies, the contributions of this means of circulation of scientific knowledge are analyzed, in the consolidation of the Research Group Representations and Scientific Concepts. The work carried out focuses both

\section{Ricardo Andrés Franco Moreno* Rómulo Gallego Badillo** Royman Pérez Miranda ${ }^{\star \star *}$}

Magíster en docencia de la química (upn) y doctorando en ciencias de la educación (uptc), profesor del Departamento de Química - Grupo irec, Universidad Pedagógica Nacional, Bogotá, Colombia.rfranco@pedagogica.edu.co

** Magíster en educación (upn) y profesor emérito del Departamento de Química - Grupo irec, Universidad Pedagógica Nacional, Bogotá, Colombia.rgallego@pedagogica.edu.co

*** Magíster en docencia de la química (upn) y profesor del Departamento de Química - Grupo irec, Universidad Pedagógica Nacional, Bogotá, Colombia.royman@pedagogica.edu.co 
on the tracking of the contributions undertaken by this academic group during the validity of the journal - characterizing the main lines of research, thematic areas, methodologies, among other aspects -, as in the analysis of citations that the community of specialists has done on some of these articles. It is concluded that, celebrating 25 years of existence, this journal represents a fundamental scenario in the development and dynamics of academic groups such as the one reported here, at the institutional, local, national and Latin American level.

Keywords

TED journal; IREC Group; didactics of experimental sciences; documentary research; scientometrics

\section{Resumo}

Por ocasião da comemoração dos 25 anos da revista Tecné, Episteme e Didaxis - TED, este artigo comunica os resultados de uma investigação histórico-documental na qual, do ponto de vista metodológico dos estudos cienciometricos, são analisadas as contribuições deste veículo de circulação do conhecimento científico, na consolidação do Grupo de Pesquisa em Representações e Conceitos Científicos. O trabalho realizado enfoca tanto o rastreamento das contribuições desse grupo acadêmico durante a validade da revista - caracterizando as principais linhas de pesquisa, áreas temáticas, metodologias, entre outros aspectos -, como na análise de citações que a comunidade de especialistas já fez em alguns desses artigos. Conclui-se que, comemorando 25 anos de existência, esta revista representa um cenário fundamental no desenvolvimento e na dinâmica de grupos acadêmicos como o aqui relatado, em nível institucional, local, nacional e latino-americano.

Palavras-chave

Revista TED; Grupo IREC; didática das ciências experimentais; pesquisa documental; cienciometria 


\section{Introducción}

Antes de la publicación de La estructura de las revoluciones científicas (1962) de T. S. Kuhn (1922-1996), con la versión castellana de 1971, en la que establece una concepción sobre la construcción de las ciencias de la naturaleza, diferente a la que circulaba en el aparato escolar y en los libros de textos, se postulaba que esa construcción había obedecido a la genialidad de hombres y mujeres que habían nacido con una dote intelectual especial para dedicarse a esta labor. Ni siquiera se hacía hincapié en la influencia del sistema educativo y de los educadores que habían realizado el trabajo pedagógico y didáctico que los inclinó y estimuló en esa dirección. El concepto psicológico de genio se apoderó de la mentalidad de los escolares y, puesto que algunos de sus nombres y apellidos eran de origen alemán o inglés, en especial, se propagó la creencia de que se debía tener la tez blanca y el cabello rubio, para acceder al entendimiento de la naturaleza de esas ciencias.

Kuhn, desde una revisión histórica, conceptuó que ese trabajo había sido adelantado por comunidades científicas, dentro de los diferentes paradigmas que cada una elaboraba para orientar su trabajo de producción de conocimientos. Cada paradigma era conservado y defendido de manera celosa, hasta cuando no podía explicar un hecho determinado, ni con la introducción de ajustes conceptuales y metodológicos. La comunidad entraba en una crisis paradigmática y se veía en la necesidad de un cambio paradigmático, esto es, de formular uno nuevo. De esta manera enseñó que el desarrollo del conocimiento científico obedecía a esta dinámica cognoscitiva.

El conocimiento científico es, entonces, producto de una labor colectiva e histórica, en la cual, además, la comunidad científica asume el compromiso de formar a las nuevas generaciones de practicantes, a quienes también introducen en ella, enseñándoles a escribir artículos. Para esta introducción primero los escriben los científicos reconocidos, quienes colocan a los noveles en una posición después de ellos. Luego, en una segunda publicación encabezan la lista de autores, y, finalmente, ya reconocidos los nuevos, pueden escribir y firmar sus propios artículos. Escrito de esta manera parece un proceso fácil, pero no es así. El editor de una revista especializada cuenta con un comité editorial que se encarga de recibir y evaluar de forma interna las propuestas, evaluación que les asigna una calificación, la cual si es negativa hace que sean rechazadas $y$, en caso contrario, son remitidas a por lo menos tres especialistas para que sean ellos quienes den el veredicto definitivo.

Esos evaluadores externos, especialistas en la temática que aborda la propuesta, a su vez, pueden rechazarla o aceptarla bajo la condición de que los autores introduzcan una serie de correcciones tanto conceptuales como metodológicas, y sugerencias sobre las referencias y la bibliografía. El editor reenvía estas críticas y sugerencias a los autores para que ellos, de aceptarlas, prácticamente reescriban el artículo. Este proceder rige, incluso, para científicos reconocidos, a quienes les han devuelto propuestas de aquellas revistas en las que con anterioridad les han publicado artículos de cierta relevancia (Hoffmann, 1997).

Por otro lado, y desde la perspectiva de la cienciometría, se estima que científico es aquella persona a quien una revista especializada y de prestigio, le ha aceptado y publicado por lo menos una colaboración en sus páginas. Pero no basta con este hecho, pues se exige que su artículo sea referenciado por otros investigadores en revistas diferentes y del mismo prestigio (Barona, 1994). Lo anterior significa que no es solo el título académico ni la investigación en sí misma los que determinan 
que alguien sea considerado como científico, ya que, para tal efecto, tiene que aparecer, hoy, en las páginas electrónicas. Ser científico va, entonces, más allá de las especializaciones y doctorados obtenidos los cuales, si sus portadores no tienen el reconocimiento dado por sus pares, solo sirven para colgarlos en las salas o en la biblioteca de la residencia.

\section{Antecedentes}

Frente al impacto e incidencia de las publicaciones realizadas en revistas científicas, libros y handbooks especializados en el campo de la didáctica de las ciencias experimentales, es preciso reconocer que la comunidad de investigadores en este campo del conocimiento ha venido mostrando un especial interés. En el ámbito global, Fraser, Tobin y McRobie (2012), pusieron a disposición de esta comunidad de especialistas la segunda versión del Second international handbook of science education el cual es producto de un notable acopio de producciones académicas que, iniciada la segunda década del siglo XXI, revisten importantes aportes conceptuales y metodológicos en temáticas como: currículo, naturaleza de la ciencia, políticas educativas, evaluación, historia y epistemología de las ciencias experimentales, entre otras.

Puntualizando en las revistas especializadas en didáctica de las ciencias experimentales, tras el balance documental presentado por Gil Pérez (1994), en el que se evalúa una década de investigación en didáctica de las ciencias experimentales, en lberoamérica se generó un movimiento de producciones académicas dedicadas a la evaluación cualitativa y cuantitativa de la producción de conocimiento en el área, en especial, tomando como referente, por su carácter dinamizador de las comunidades científicas, las publicaciones en revistas especializadas. Desde esta perspectiva, Gallego Badillo (2008) en su calidad de director de la revista TED, concitó a editores de revistas de diferentes países en un encuentro internacional de revistas científicas dedicadas a la educación, la pedagogía y la didáctica. Se presentaron diversos manuscritos que en su mayoría fueron el producto de ejercicios bibliométricos, cienciométricos y documentales de literatura científica.

En el caso particular de la revista TED, Soler y Parga (2008) realizaron un balance de las tendencias investigativas y principales temáticas sobre las que en esta revista se publicaron artículos en un periodo de 5 años, para lo cual emplearon técnicas documentales y bibliométricas. De igual manera, la revista ha sido objeto de análisis en investigaciones históricas y documentales acerca de líneas de investigación en didáctica de las ciencias experimentales, como las relaciones CTSA (Garzón, Zúñiga y Franco, 2011) y los Trabajos Prácticos de Laboratorio - TPL en la enseñanza de las ciencias (Franco, Velasco y Riveros, 2017), entre otras.

Frente a las investigaciones específicas adelantadas por el Grupo IREC desde la perspectiva del estudio de las producciones científicas, por una parte, en su reconstrucción histórica acerca de las investigaciones en didáctica de las ciencias financiadas por Colciencias, Fuentes (2005) adoptó como criterio la existencia 
de publicaciones en revistas indexadas tanto del país como internacionales. Por otra, en el grupo se documentó la producción de la comunidad científica colombiana en disciplinas como química, física y biología y su presencia internacional a partir de un análisis cienciométrico de revistas de amplio reconocimiento a nivel nacional: Revista Colombiana de Química, Revista Colombiana de Física y Actualidades Biológicas; y mundial: Journal of the American Chemical Society, American Journal of Physics y Journal of Experimental Biology (Franco Moreno, Gallego Badillo y Pérez Miranda, 2009). Dichos estudios coinciden en dar cuenta de que, el análisis de la literatura científica permite establecer relaciones entre la dinámica de la producción de nuevo conocimiento en programas de formación de profesores de ciencias, así como en los grupos de investigación, asociaciones científicas, entidades estatales y privadas, y en suma, en las comunidades científicas.

\section{Marco conceptual}

\section{La didáctica de las ciencias experimentales como disciplina científica}

Es propio de la tradición en la comunidad de profesores y de sus formadores considerar la didáctica como la parte instrumental de la pedagogía. Concepción no gratuita y producto de esa tradición sesgada por una versión positivista de la actividad relacionada con la formación de las nuevas generaciones, especialmente en ciencias. En ese mismo marco ha hecho carrera la concepción de que "basta con saber una ciencia para enseñarla" por lo que todos los esfuerzos en la formación de profesores se dirigen a la consolidación de ese saber y se le otorga licencia a quienes se forman en ese campo para ejercer su docencia.
El intento de comprender el arraigo de esta concepción obliga una revisión histórica de la misma, como lo demuestran diferentes investigaciones entre ellas la realizada por Gallego Badillo, Pérez Miranda, Torres de Gallego y Amador Rodríguez (2004). Basados en esta investigación y como es conocido, en la antigua Grecia se diferenciaban las artes nobles propias de la aristocracia de las innobles ejercidas por los esclavos, estas últimas que se aprendían por imitación y con escasa demanda intelectual. Tales artes innobles, en Roma, las practicaban además los no ciudadanos, quienes fueron organizados por el Emperador Numa en corporaciones de artesanos, de las cuales surgieron los "Collegia artíficum". Para enseñar el oficio en ellos había que conocerlo y ser reconocido como maestro artesano por la sociedad en general, el aprendizaje se hacía por observación e imitación de sus maestros. La propuesta de Martín Lutero de la Reforma en el siglo xv demandaba de todos el saber leer y escribir para la interpretación de la doctrina, por lo que fue necesaria una educación y una escuela generalizadas.

En este trasegar de la didáctica aporta a su constitución Calvino con la introducción a la "pedagogía" asociada a la educación de los infantes y F. Ratke propone la didáctica como discusión centrada en el estudio de la eficiencia de los métodos de enseñanza. Juan Amos Comenio (1657), con su "Didáctica Magna" la focaliza en "el arte de enseñar todo a todos con ahorro de tiempo y fatiga", que trascendió a la regulación por parte de las autoridades educativas para exigir capacitación y entrenamiento a los enseñantes sobre lo que han de enseñar y cómo han de hacerlo. Esta obligación condicionó a los profesores a la función de operarios de los sistemas educativos, dado que según Comenio los enseñantes no se han de encargar de estos menesteres ya que de ello se encargarían otros y, en consecuencia, los 
enseñantes han de responder por lo que se les encarga y de cómo hacerlo. Es F. J. Herbart quien inicia la discusión sobre la pedagogía como ciencia fundada en la filosofía y la psicología y, por su parte, E. Durkheim y A. Comte se inclinan por la sociología y la psicología como las ciencias de la educación, con lo que la didáctica continuaría con la versión de Comenio.

Ya en el siglo xx la conmoción causada por el lanzamiento del Sputnik, en 1957, por la Unión Soviética y su implicación en el dominio científico y tecnológico en el mundo, surge en Estados Unidos la necesidad de una ciencia para todos con el fin de potenciar el número de estudiantes que se dedicaran a la investigación científica. Emergen propuestas distintas, entre ellos los programas alfabeto centrados en el llamado "método científico" desde la aproximación empiropositivista que derivó a la llamada tecnología educativa. Esta perspectiva, fundada en el conductismo operante, propone una enseñanza por objetivos. Con los cambios en la concepción de ciencia y de práctica científica, promovidos por T. S. Kuhn (1972), K. Popper (1962), I. Lakatos (1983) en diferentes países se consideró abordar, desde la didáctica, el problema de la enseñanza de las ciencias como un problema de construcción teórica. Un campo de investigación científica estaba a la vista. Así, Novak (1988), Aliberas, Gutiérrez e lzquierdo (1989), Gil, Carrascosa y Martínez-Terrades (1999), Sanmartí e lzquierdo (2001), Adúriz-Bravo, (2000), entre otros muchos, asumen la didáctica de las ciencias como una disciplina científica fundamentada de modo conceptual, con una comunidad científica que ha delimitado unos campos de conocimiento y desde los cuales se adelantan sus investigaciones, programas de formación inicial y de posgrado en el campo, revistas especializadas que publican la producción de la comunidad, congresos y eventos en los que se discuten las tendencias futuras de esa didáctica y los problemas por abordar.

En la actualidad, se consideran los campos de conocimiento e investigación de la didáctica de las ciencias de la naturaleza, la enseñabilidad y la enseñanza de las ciencias como problema didáctico; las concepciones alternativas de los estudiantes; las concepciones de los profesores de ciencias; la confiabilidad de los textos de enseñanza; la evaluación en ciencias como problema didáctico; la formación inicial y continua de profesores de ciencias; historia, epistemología y didáctica de las ciencias y otros campos que van surgiendo dada la dinámica investigativa que le imprime una comunidad ávida por responder preguntas sobre el trabajo en el aula, la producción de conocimiento científico y su relación con la tecnología y sociedad en general.

\section{Congresos científicos, revistas especializadas y cienciometría}

Después del Congreso de Karlsruhe (Gallego Badillo, Gallego Torres y Pérez Miranda, 2012), se generalizó que todas las comunidades de especialistas se congregaran periódicamente, con el fin de examinar de forma crítica el estado de desarrollo y las direcciones que era necesario tomar en su disciplina, para 
responder tanto a los retos internos como a los externos planteados por las perspectivas científicas y tecnológicas, esperados por la clase de sociedad, que ese desarrollo había impulsado. Se constituyó también en una praxis para que los noveles conocieran, identificaran y se relacionaran con los personajes reconocidos por sus aportes, invitados como conferencistas centrales en dichos congresos. De hecho, los organizadores eran amigos de esos conferencistas, además de ser los profesores de esta nueva generación.

Los congresos se convirtieron, entonces, en un mecanismo de reproducción, y también de selección de cada comunidad científica. Así, y de conformidad con los intereses y las aspiraciones de los noveles, desde las primeras aproximaciones su meta fue la pertenencia al colectivo científico de su elección. Dentro de una apreciación general, en ellos ha habido siempre aquellos quienes solo aspiran a obtener un título profesional, para llevar una vida plácida lejos de los conflictos y luchas que conllevan la aspiración a ser un científico reconocido en el ámbito internacional. En dicha arena devienen también las revistas científicas especializadas (Gallego Badillo, 2008).

La sociedad del conocimiento en la que vivimos, desde hace poco menos de cuatro décadas, ha venido experimentando profundas transformaciones en la forma en la que los resultados de nuevas investigaciones son decodificados, editados y presentados como producto de nuevos conocimientos en forma de artículos científicos. Entre dichos cambios, agenciados en su mayoría por la vertiginosa revolución de las infotecnologías (Pla I Brunet, 2003), se encuentran el aumento exponencial en el número de revistas especializadas, bases de datos y plataformas de indexación, buscadores web y en suma, un incremento considerable del número de publicaciones.
Ese aumento en la cantidad de publicaciones, además de fortalecer aquella premisa de que pertenece a una comunidad científica quien publica artículos en revistas especializadas y que, en consecuencia, ha de catalogarse de conocimiento científico el contenido que circula en los artículos (Barona, 1994), robusteció también la cienciometría, entendida esta como una disciplina emergente que, partiendo de modelos, razonamientos y métricas de orden matemático y estadístico, permite medir y cuantificar buena parte de los resultados de la actividad científica (Kragh, 2007). De este modo, la cantidad de artículos por autor, el análisis de las citaciones de estas producciones realizadas or otros autores, la colaboración nacional e internacional, las instituciones patrocinantes, los países y regiones, el género, las diversas clasificaciones y perspectivas analíticas de la literatura publicada, entre otros, se constituyeron en indicadores cienciométricos (Franco, Velasco y Riveros, 2017).

En esta lógica, la cienciometría contribuyó también al desarrollo y definición de criterios técnicos como la indexación de las revistas, su factor de impacto y el denominado

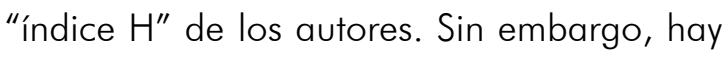
que puntualizar que, tanto las métricas como los parámetros cienciométricos han recibido múltiples revisiones y lecturas críticas desde campos científicos como el de la sociología de la ciencia, al cuestionar con profundidad, desde los procesos y dinámicas de producción de los artículos, hasta la capacidad de dichos parámetros para evaluar la apropiación y usos sociales del conocimiento científico, las entidades y personas que lo agencian (Golombek, 2007). No obstante, en esta investigación documental se vinculan criterios cienciométricos que contribuyen de manera significativa a los análisis emprendidos. 


\section{Metodología}

Partiendo de los fundamentos conceptuales de la bibliometría y de la cienciometría aplicados a la investigación documental en didáctica de las ciencias experimentales (Franco Moreno, Gallego Badillo y Pérez Miranda, 2009), se seleccionó la revista Tecné, Episteme y Didaxis - TED, como una publicación de amplio reconocimiento y de alto impacto para la comunidad de investigadores en didáctica de las ciencias experimentales de Suramérica. En la tabla 1 se presentan los datos formales de la revista:

Tabla 1. Ficha de información revista ted

\begin{tabular}{|l|l|}
\hline Nombre de la revista & Tecné, Episteme y Didaxis - TED \\
\hline País & Colombia \\
\hline ISSN & $0121-3814$ \\
\hline Link del sitio web & http://revistas.pedagogica.edu.co/index.php/TED \\
\hline Indexación en Publindex de Colciencias & B \\
\hline Bases de datos & ERA, Latindex, IRESIE, SCIELO, Ulrich's, Dialnet, Clase, REDIB, DOAJ \\
\hline
\end{tabular}

Fuente: elaboración propia, con base al sitio web de la revista.

\section{Resultados y análisis}

Luego de acopiar la colección acumulada en los 25 años de vigencia de la revista (sin incluir editoriales, los números extraordinarios publicados en el marco de eventos académicos promovidos por la revista, ni la sección de reseñas de tesis de maestría), se hizo el rastreo de aquellas contribuciones realizadas por integrantes del Grupo de Investigación IREC, codificándolas y clasificándolas de acuerdo con lo propuesto por Franco, Velasco y Riveros (2017), en líneas de investigación, áreas temáticas, metodologías y tipología de los artículos, entre otros aspectos.

De conformidad con las líneas de investigación en didáctica de las ciencias experimentales, desde las cuales el Grupo IREC ha desarrollado su actividad investigativa (Franco Moreno, Gallego Badillo y Pérez Miranda, 2015), líneas que concuerdan con caracterizaciones de campos de investigación en el área realizadas por otros colectivos académicos (Nardi, 2003; Parga, 2007; Zambrano, Salazar, Candela y Villa, 2017), estos artículos fueron objeto de la siguiente clasificación en las líneas de investigación: Formación inicial y continua de profesores de ciencias experimentales - FICPC; Relaciones entre historia, epistemología y didáctica de las ciencias - RHEDC; Confiabilidad en textos de enseñanza y transposición didáctica - CTETD; Relaciones entre enseñanza y aprendizaje de las ciencias experimentales - REACE. 
Al analizar el contenido de los artículos, su tipología se clasificó en: reflexión-revisión: "Revref"; investigación: "Inv"; y en cuanto a su metodología en: cualitativa: "cuali"; cuantitativa: "cuanti" y en el caso de métodos mixtos: "mix". De igual manera, dada la importancia del indicador cienciométrico de colaboración, la cual se puede catalogar como: grupal (G), institucional $(\mathrm{It})$, regional $(\mathrm{R})$, nacional $(\mathrm{N})$ e internacional $(\mathrm{In})$, como se muestra en la tabla 2 :

Tabla 2. Artículos publicados en revista TED por autores integrantes del Grupo IREC

\begin{tabular}{|c|c|c|c|}
\hline Cód & Título del artículo. Número y año & Autor(es) / (colaboración) & $\begin{array}{l}\text { Tipología/ } \\
\text { Metodología } \\
\text { Línea de inv }\end{array}$ \\
\hline 1 & $\frac{\text { Contribución del cómic a la imagen de la ciencia }}{7,2000}$ & $\begin{array}{l}\text { Daniel Gil Pérez, Jaime Carrascosa Alís, } \\
\text { Adriana Patricia Gallego e Isabel Fernández (In) }\end{array}$ & $\begin{array}{l}\text { Inv } \\
\text { Cuali } \\
\text { RHEDC }\end{array}$ \\
\hline 2 & $\begin{array}{l}\text { Una aproximación al aprendizaje total del } \\
\text { concepto de reacción química } \\
7,2000\end{array}$ & $\begin{array}{l}\text { Cheyron Castellanos Sánchez y Rómulo } \\
\text { Gallego Badillo (lt) }\end{array}$ & $\begin{array}{l}\text { Inv } \\
\text { Cuali } \\
\text { REACE }\end{array}$ \\
\hline 3 & $\begin{array}{l}\text { El concepto pedagógico y didáctico de ámbito en } \\
\text { la enseñanza de la química } \\
7,2000\end{array}$ & $\begin{array}{l}\text { Royman Pérez Miranda y Rómulo Gallego } \\
\text { Badillo (G) }\end{array}$ & $\begin{array}{l}\text { Rev-ref } \\
\text { REACE }\end{array}$ \\
\hline 4 & $\begin{array}{l}\text { Competencias cognoscitivas y evaluación } \\
8,2000\end{array}$ & $\begin{array}{l}\text { Royman Pérez Miranda y Rómulo Gallego } \\
\text { Badillo }(G)\end{array}$ & $\begin{array}{l}\text { Rev-ref } \\
\text { REACE }\end{array}$ \\
\hline 5 & $\begin{array}{l}\text { El sentido de la pedagogía y la didáctica en las } \\
\text { tecnologías } \\
8,2000\end{array}$ & $\begin{array}{l}\text { José Ocampo Rodríguez y Rómulo Gallego } \\
\text { Badillo (It) }\end{array}$ & $\begin{array}{l}\text { Rev-ref } \\
\text { RHEDC }\end{array}$ \\
\hline 6 & $\begin{array}{l}\text { ¿Nuevos horizontes de investigacion? } \\
10,2001\end{array}$ & Rómulo Gallego Badillo (G) & $\begin{array}{l}\text { Rev-ref } \\
\text { RHEDC }\end{array}$ \\
\hline 7 & $\begin{array}{l}\text { El efecto didáctico en ciencias experimentales } \\
10,2001\end{array}$ & $\begin{array}{l}\text { Rómulo Gallego Badillo, Royman Pérez } \\
\text { Miranda, Luz Nery Torres de Gallego y María } \\
\text { Nelly Mantilla Mantilla (R) }\end{array}$ & $\begin{array}{l}\text { Rev-ref } \\
\text { FICPC }\end{array}$ \\
\hline 8 & $\begin{array}{l}\text { El aprendizaje total de los conceptos científicos } \\
\text { ácido-base } \\
10,2001\end{array}$ & Sara Zafra A. (It) & $\begin{array}{l}\text { Inv } \\
\text { Mixta } \\
\text { REACE }\end{array}$ \\
\hline 9 & $\begin{array}{l}\text { Historia de la didáctica de las ciencias: un campo } \\
\text { de investigación } \\
12,2002\end{array}$ & $\begin{array}{l}\text { Rómulo Gallego Badillo, Adriana Patricia } \\
\text { Gallego Torres yRoyman Pérez Miranda (G) }\end{array}$ & $\begin{array}{l}\text { Rev-ref } \\
\text { RHEDC }\end{array}$ \\
\hline 10 & $\begin{array}{l}\text { Entendimiento del concepto mol logrado por los } \\
\text { estudiantes en un curso de química general de } \\
\text { corte constructivista } \\
13,2003\end{array}$ & $\begin{array}{l}\text { Carlos Utria Echeverría, Roberto Figueroa } \\
\text { Molina (R) }\end{array}$ & $\begin{array}{l}\text { Inv } \\
\text { Mixta } \\
\text { FICPC }\end{array}$ \\
\hline 11 & $\begin{array}{l}\text { Estudio histórico-epistemológico del modelo } \\
\text { atómico de Rutherford } \\
14,2003\end{array}$ & María Victoria Uribe y Luigi Cuellar (It) & $\begin{array}{l}\text { Inv } \\
\text { Mixta } \\
\text { RHEDC }\end{array}$ \\
\hline 12 & $\begin{array}{l}\text { Estudio histórico-epistemológico sobre la teoría } \\
\text { o modelo de la estructura química del ácido } \\
\text { desoxirribonucleico (ADN) } \\
14,2003\end{array}$ & $\begin{array}{l}\text { Angélica María García Torres } \\
(\mathrm{It})\end{array}$ & $\begin{array}{l}\text { Inv } \\
\text { Cuali } \\
\text { RHEDC }\end{array}$ \\
\hline 13 & $\begin{array}{l}\text { Estudio exploratorio de las interacciones mentales } \\
\text { de los estudiantes de sexto grado sobre el } \\
\text { entendimiento conceptual de multiplicación } \\
15,2004\end{array}$ & $\begin{array}{l}\text { Roberto Figueroa Molina, Carlos Utria } \\
\text { Echeverría, Rafael Colpas Castillo, Antonio } \\
\text { Araújo Pereira (N) }\end{array}$ & $\begin{array}{l}\text { Inv } \\
\text { Mixta } \\
\text { FICPC }\end{array}$ \\
\hline 14 & $\begin{array}{l}\text { Estudio inicial de dos programas para la } \\
\text { formación de Licenciados en Química } \\
\text { 16, } 2004\end{array}$ & $\begin{array}{l}\text { Rafael Yecid Amador Rodríguez y Rómulo } \\
\text { Gallego Badillo }(G)\end{array}$ & $\begin{array}{l}\text { Inv } \\
\text { Mixta } \\
\text { FICPC }\end{array}$ \\
\hline
\end{tabular}




\begin{tabular}{|c|c|c|c|}
\hline & & & Línea de inv \\
\hline 15 & $\begin{array}{l}\text { Análisis de la transposición didáctica de los } \\
\frac{\text { conceptos de calor y temperatura en los libros de }}{17,2005}\end{array}$ & $\begin{array}{l}\text { Johanna Patricia Camacho González y Royman } \\
\text { Pérez Miranda (G) }\end{array}$ & $\begin{array}{l}\text { Inv } \\
\text { Cuali } \\
\text { CTETD }\end{array}$ \\
\hline 16 & $\begin{array}{l}\text { Entendimiento conceptual de estudiantes } \\
\text { universitarios sobre conceptos en las ciencias } \\
\text { experimentales } \\
18,2005\end{array}$ & $\begin{array}{l}\text { Roberto Figueroa Molina, Carlos Utria } \\
\text { Echeverría y Rafael Colpas Castillo (N) }\end{array}$ & $\begin{array}{l}\text { Inv } \\
\text { Mixta } \\
\text { FICPC }\end{array}$ \\
\hline 17 & $\begin{array}{l}\text { Consideraciones sobre la categoría } \\
\text { epistemológica de modelo } \\
19,2006\end{array}$ & Adriana Patricia Gallego Torres (G) & $\begin{array}{l}\text { Rev-ref } \\
\text { RHEDC }\end{array}$ \\
\hline 18 & $\begin{array}{l}\text { Los conceptos sobre ciencia y trabajo científico. } \\
\frac{\text { Sus implicaciones en la elaboración de los }}{20,2006}\end{array}$ & $\begin{array}{l}\text { Silvio F. Daza Rosales y José R. Arrieta Vergara } \\
\text { (N) }\end{array}$ & $\begin{array}{l}\text { Inv } \\
\text { Mixta } \\
\text { RHEDC }\end{array}$ \\
\hline 19 & $\begin{array}{l}\text { Un análisis histórico-epistemológico de los } \\
\text { trabajos de D. Mendéleiev sobre la periodicidad } \\
\text { química } \\
20,2006\end{array}$ & $\begin{array}{l}\text { Fredy Garay Garay, Rómulo Gallego Badillo y } \\
\text { Royman Pérez Miranda (G) }\end{array}$ & $\begin{array}{l}\text { Inv } \\
\text { Cuali } \\
\text { RHEDC }\end{array}$ \\
\hline 20 & $\begin{array}{l}\frac{\text { Ciencia, historia, epistemología y didáctica de las }}{\text { Ciencias: las comunidades de especialistas }} \\
22,2007\end{array}$ & Adriana Patricia Gallego Torres (G) & $\begin{array}{l}\text { Rev-ref } \\
\text { RHEDC }\end{array}$ \\
\hline 21 & $\begin{array}{l}\text { Una construcción histórico-epistemológica del } \\
\text { modelo del octeto para el enlace químico } \\
23,2008\end{array}$ & $\begin{array}{l}\text { Sulma Urbina Duarte, Rómulo Gallego Badillo, } \\
\text { Royman Pérez Miranda y Adriana Patricia } \\
\text { Gallego Torres (R) }\end{array}$ & $\begin{array}{l}\text { Inv } \\
\text { Cuali } \\
\text { RHEDC }\end{array}$ \\
\hline 22 & $\begin{array}{l}\text { Desde qué versiones epistemológicas construyen } \\
\text { modelos mentales los profesores en formación } \\
\frac{\text { inicial: una investigación didáctica }}{24,2008}\end{array}$ & $\begin{array}{l}\text { Rafael Yecid Amador Rodríguez, Rómulo } \\
\text { Gallego Badillo y Royman Pérez Miranda (G) }\end{array}$ & $\begin{array}{l}\text { Inv } \\
\text { Mixta } \\
\text { RHEDC }\end{array}$ \\
\hline 23 & $\begin{array}{l}\text { Modelos científicos y algunas implicaciones en la } \\
\text { formación inicial de profesores de química } \\
25,2009\end{array}$ & $\begin{array}{l}\text { Quira A. Sanabria Rojas, Royman Pérez } \\
\text { Miranda y Rómulo Gallego Badillo (G) }\end{array}$ & $\begin{array}{l}\text { Inv } \\
\text { Cuali } \\
\text { FICPC }\end{array}$ \\
\hline 24 & $\begin{array}{l}\text { La matematización de los procesos químicos. } \\
\text { Primera parte } \\
27,2010\end{array}$ & $\begin{array}{l}\text { Rómulo Gallego Badillo, Royman Pérez } \\
\text { Miranda y Roberto Figueroa Molina (N) }\end{array}$ & $\begin{array}{l}\text { Rev-ref } \\
\text { REACE }\end{array}$ \\
\hline 25 & $\begin{array}{l}\text { Matematización de los procesos químicos. } \\
\text { Segunda parte } \\
28,2010\end{array}$ & $\begin{array}{l}\text { Rómulo Gallego Badillo, Royman Pérez } \\
\text { Miranda y Roberto Figueroa Molina (N) }\end{array}$ & $\begin{array}{l}\text { Rev-ref } \\
\text { REACE }\end{array}$ \\
\hline 26 & $\begin{array}{l}\text { Los trabajos prácticos de laboratorio en la } \\
\text { enseñanza de las ciencias: tendencias en revistas } \\
\text { especializadas (2012-2016) } \\
41,2017\end{array}$ & $\begin{array}{l}\text { Ricardo Andrés Franco, María Velasco y Carlos } \\
\text { Riveros (G) }\end{array}$ & $\begin{array}{l}\text { Inv } \\
\text { Mixta } \\
\text { REACE }\end{array}$ \\
\hline
\end{tabular}

Fuente: elaboración propia

Los títulos de artículos subrayados, como se enuncia más adelante, son los de mayor citación en producciones escriturales posteriores.

La anterior información se resume en las tablas 3 y 4 : 
Tabla 3. Artículos publicados por Grupo irec en revista ted por número y año

\begin{tabular}{|c|c|c|c|c|c|c|c|}
\hline No. & Año & $\begin{array}{c}\text { Cant. } \\
\text { Artículos }\end{array}$ & $\begin{array}{c}\text { Art. Grupo } \\
\text { IREC }\end{array}$ & No. & Año & $\begin{array}{l}\text { Cant. } \\
\text { Artículos }\end{array}$ & $\begin{array}{l}\text { Art. Grupo } \\
\text { IREC }\end{array}$ \\
\hline 1 & 1991 & 7 & 0 & 22 & 2007 & 9 & 1 \\
\hline 2 & 1992 & 9 & 0 & 23 & 2008 & 9 & 1 \\
\hline 3 & 1998 & 6 & 0 & 24 & 2008 & 9 & 1 \\
\hline 4 & 1998 & 13 & 0 & 25 & 2009 & 6 & 1 \\
\hline 5 & 1999 & 10 & 0 & 26 & 2009 & 8 & 0 \\
\hline 6 & 1999 & 13 & 0 & 27 & 2010 & 8 & 1 \\
\hline 7 & 2000 & 9 & 3 & 28 & 2010 & 7 & 1 \\
\hline 8 & 2000 & 13 & 2 & 29 & 2011 & 7 & 0 \\
\hline 9 & 2001 & 13 & 0 & 30 & 2011 & 7 & 0 \\
\hline 10 & 2001 & 12 & 3 & 31 & 2012 & 6 & 0 \\
\hline 11 & 2002 & 6 & 0 & 32 & 2012 & 9 & 0 \\
\hline 12 & 2002 & 9 & 1 & 33 & 2013 & 7 & 0 \\
\hline 13 & 2003 & 11 & 1 & 34 & 2013 & 6 & 0 \\
\hline 14 & 2003 & 10 & 2 & 35 & 2014 & 6 & 0 \\
\hline 15 & 2004 & 9 & 1 & 36 & 2014 & 6 & 0 \\
\hline 16 & 2004 & 8 & 1 & 37 & 2015 & 6 & 0 \\
\hline 17 & 2005 & 8 & 1 & 38 & 2015 & 10 & 0 \\
\hline 18 & 2005 & 9 & 1 & 39 & 2016 & 7 & 0 \\
\hline 19 & 2006 & 9 & 1 & 40 & 2016 & 10 & 0 \\
\hline 20 & 2006 & 7 & 2 & 41 & 2017 & 11 & 1 \\
\hline 21 & 2007 & 8 & 0 & 42 & 2017 & 10 & 0 \\
\hline \multicolumn{8}{|c|}{ Total artículos revista TED: 363} \\
\hline
\end{tabular}

Fuente: elaboración propia

Tabla 4. Resumen clasificación de los artículos según tipología y metodología

\begin{tabular}{lcccc}
\multicolumn{1}{c}{$\begin{array}{c}\text { Tipología y metodología } \\
\text { de las contribuciones }\end{array}$} & Cant. & \multicolumn{1}{c}{$\begin{array}{c}\text { Tipo de colaboración } \\
\text { de las contribuciones }\end{array}$} & Cant. \\
\hline Artículos de revisión-reflexión & 10 & Grupal & 12 \\
\hline Artículos de investigación & 16 & Institucional & 5 \\
\hline Metodología cualitativa & 7 & Regional & 3 \\
\hline Metodología cuantitativa & 0 & Nacional & 5 \\
\hline Metodología mixta & 9 & Internacional & 1
\end{tabular}

Fuente: elaboración propia 
Como se aprecia, de la totalidad de artículos publicados en la revista TED hasta su número 42 (363), el Grupo de investigación IREC ha aportado 26 manuscritos, cuya mayoría (25) fueron publicados durante el periodo 2000-2010, lo cual puede atribuirse al especial impulso que -en un trabajo mancomunado de quienes en esta etapa fueran director, el profesor Rómulo Gallego Badillo, y codirector del Grupo IREC, Royman Pérez Miranda- se dio para que los grupos de investigación de la Facultad de Ciencia y Tecnología de la UPN contribuyeran al fortalecimiento de la revista en la época. De igual manera, esto obedece al empeño decidido del Grupo IREC por presentar proyectos de investigación ante instancias como el Centro de Investigaciones CIUP y Colciencias para su financiación, lo cual permitió la vinculación de profesores en formación posgradual e inicial, cuyas producciones se ven reflejadas en las cifras que se presentan.

Las contribuciones realizadas por el Grupo IREC a la revista TED son en SU mayoría de investigación. De los 16 artículos con esta clasificación, que representan el 61,5\%, 9 exhiben una metodología mixta y 7 son desde diseños metodológicos cualitativos, con lo cual queda sentado que las aproximaciones metodológicas de carácter netamente cuantitativo no han sido el interés en la tradición investigativa del Grupo IREC, cuya actividad científica ha tomado distancia de la aproximación epistemológica positivista, inclinándose más bien por perspectivas como la constructivista moderada y adoptando algunas premisas del paradigma sociocrítico en la enseñanza de las ciencias (Franco Moreno, Gallego Badillo y Pérez Miranda, 2015).

De ello da cuenta el indicador de colaboración, de una alta importancia en los estudios cienciométricos e históricos de la documentación científica (Kragh, 2007), pues 12 artículos son desarrollados específicamente dentro del grupo con una buena participación de estudiantes de posgrado; 5 reflejan una colaboración institucional evidenciada en la participación de profesores de diferentes programas o instituciones locales; otros 5 son en colaboración regional; 3 lo son con colegas de otras instituciones a nivel nacional y 1 en colaboración internacional. Al respecto de lo último habría que puntualizar, por una parte, que las publicaciones conjuntas con investigadores de lberoamérica fueron tramitadas en revistas internacionales, así como en libros y en capítulos de libros (Gallego Badillo, Pérez Miranda y Franco Moreno, 2016), y por otra, que fue en el periodo 2000-2010 cuando se fomentó una política editorial para la revista TED, en la que se prestó especial atención a la consolidación de un comité científico-asesor de talla internacional, lo cual cautivó el interés de reconocidos investigadores en el campo de la didáctica de las ciencias experimentales por publicar en la revista, a la vez que dicho carácter internacional se dinamizó con la creación del Congreso Internacional sobre Formación de Profesores de Ciencias, cuya octava versión fue celebrada ya en el 2018, y ha venido en un afianzamiento permanente con la participación activa del grupo en la Red Latinoamericana de Investigación en Didáctica de las Ciencias Experimentales, así como en la Rede Latinoamericana de Pesquisa em Educação Química. 
Sobre la línea Formación inicial y continua de profesores de ciencias experimentales, las 6 contribuciones encontradas representan el $23,1 \%, 5$ de estas son de investigación orientadas mayoritariamente desde diseños mixtos. Son temáticas de esta línea la formulación y desarrollo de estrategias didácticas por parte de profesores en formación inicial y avanzada en diferentes niveles educativos, así como estudios y análisis de programas, currículos y procesos de formación inicial y continua del profesorado de ciencias experimentales en Colombia.

En la línea Relaciones entre historia, epistemología y didáctica de las ciencias, se encuentra el mayor número de artículos: 12, equivalentes al 46,1\%, de los cuales 7 son de investigación y 5 de revisión-reflexión, con una distribución equilibrada entre métodos de investigación cualitativa y mixta. Las contribuciones desarrolladas en este marco se centran en temáticas tales como: reconstrucciones históricas y epistemológicas de modelos científicos en disciplinas como la química y su recontextualización en la enseñanza; análisis sobre versiones, concepciones, visiones e interpretaciones sobre ciencia y su relación con procesos didácticos; y, reflexiones acerca del carácter epistemológico de las ciencias experimentales, la didáctica de las ciencias y las comunidades científicas.

La línea Confiabilidad en textos de enseñanza y transposición didáctica es la de menor recurrencia en los artículos estudiados, con una contribución que representa el 3,8 \%, dedicada a la investigación de corte cualitativo sobre el fenómeno de la transposición didáctica de conceptos fundamentales en química como el calor y la temperatura, mediada por un libro de texto. No obstante, es necesario señalar que desde esta línea se han orientado múltiples investigaciones en el Grupo IREC, cuyas producciones académicas han sido publicadas en otras revistas de reconocido prestigio, así como en certámenes académicos especializados en el área.

En lo tocante a la línea Relaciones entre enseñanza y aprendizaje de las ciencias experimentales, las 7 contribuciones que equivalen al $26,9 \%$ tratan temáticas asociadas a la enseñanza y aprendizaje de las ciencias experimentales desde determinadas corrientes pedagógicas y modelos didácticos en el aula de clase, los cuales han dado lugar a campos de investigación en didáctica de las ciencias que han sido objeto de revisión y caracterización desde esta línea. En estas contribuciones es en donde más diversidad se encuentra en cuanto a su tipología y metodología.

\section{Un análisis de citaciones necesario}

Uno de los indicadores que coadyuvan a la consolidación tanto del factor de impacto de las revistas idexadas, como del "índice $\mathrm{H}^{\text {" de los }}$ investigadores, como ya se refirió, es el de las citaciones que los investigadores realizan de las publicaciones. En tal sentido, se hizo necesario un rastreo documental de aquellas citas que sobre las anteriores contribuciones ha realizado la comunidad de especialistas. Para tal ejercicio, se consultaron los sitios web de buscadores y bases de datos como: Google scholar, Scielo, Redalyc, Latindex y Scimago. Una vez efectuadas las búsquedas, se encontraron 57 citaciones sobre 18 de los 26 artículos antes reportados. La información se muestra en las tablas 5 y 6 : 
Tabla 5. Tipología de documentos en los que se realizan citaciones de los artículos del Grupo IREC publicados en revista TED

\begin{tabular}{|c|c|c|c|}
\hline No. & Tipo de documento & sigla & Cant \\
\hline 1 & Artículo de revista internacional & ARI & 11 \\
\hline 2 & Artículo de revista nacional & ARN & 12 \\
\hline 3 & Tesis de doctorado internacional & TDI & 3 \\
\hline 4 & Tesis de doctorado nacional & TDN & 1 \\
\hline 5 & Tesis de maestría internacional & TMl & 2 \\
\hline 6 & Tesis de maestría nacional & TMN & 15 \\
\hline 7 & Tesis de licenciatura internacional & $\mathrm{TLI}$ & 1 \\
\hline 8 & Tesis de licenciatura nacional & TLN & 2 \\
\hline 9 & Memorias de evento internacional & MEI & 7 \\
\hline 10 & Memorias de evento nacional & MEN & 1 \\
\hline 11 & Libro y capítulo de libro & LCL & 2 \\
\hline
\end{tabular}

Tabla 6. Citaciones realizadas a artículos publicados en ted, por tipo de documento

No. Art

Título y tipo del documento en el que se cita

Integración de la epistemología en la formación del profesorado de ciencias. TDI

El problema de las concepciones alternativas en la actualidad (parte III). Utilización didáctica de los errores conceptuales que aparecen en cómics, prensa, novelas y libros de texto. ARI

$1 \quad$ Ideas alternativas en conceptos científicos. ARN

Acerca del carácter tecnológico de la nueva Didáctica de las Ciencias. ARI

Influencia del contexto socio-cultural sobre las imágenes de ciencia construidas por los niños y las niñas habitantes del barrio Villa Turbay, participantes del proyecto 'la escuela busca al niño'. TLN

Validación de guías de enseñanza y aprendizaje en áreas de ingeniería de requisitos siguiendo lineamientos de SWEBOK. ARN

Aprendizaje significativo del pensamiento espacial y sistemas geométricos, integrando las TIC a través de actividades lúdicas en el primer ciclo de básica. TMN

5 Tesis-diseño de actividades de aprendizaje mediadas por TIC para la formación de docentes, en un AVA implementado en el servicio nacional de aprendizaje (SENA). TMN

Entendimiento conceptual de los estudiantes del nivel de básica secundaria sobre el concepto de ácido. ARN

Estrategia de aula para la enseñanza del concepto de equilibrio químico ácido-base para estudiantes del grado once de enseñanza media. TMN

8 Propuesta para la enseñanza del concepto ácido-base en la educación básica y media vocacional. TMN

Las nuevas tecnologías y su incidencia en la transformación de las prácticas de enseñanza de los conceptos ácido - base. TLN

Argumentación a través de la resolución de problemas para el tema la materia y sus estados de agregación. TMN

Ley periódica. Una reflexión didáctica desde la historia de las ciencias. MEI

Los grupos de investigación y la dinamización de la comunidad de especialistas. TMN 


\begin{tabular}{|c|c|}
\hline No. Art & Título y tipo del documento en el que se cita \\
\hline 10 & $\begin{array}{l}\text { El rendimiento académico en el nivel de educación media como factor asociado al } \\
\text { rendimiento académico en la universidad. ARN }\end{array}$ \\
\hline \multirow{3}{*}{11} & Radiaciones: Una mirada multidimensional. TMI \\
\hline & Voltaje, corriente y resolución de circuitos a través de las leyes de Maxwell. TMN \\
\hline & El modelo atómico de E. Rutherford. Del saber científico al conocimiento escolar. ARI \\
\hline \multirow{4}{*}{14} & $\begin{array}{l}\text { Aproximaciones y diferencias entre dos sistemas de formación inicial de profesores de } \\
\text { ciencias. ARI }\end{array}$ \\
\hline & $\begin{array}{l}\text { Los campos que hacen de la formación de profesores una línea de investigación en la } \\
\text { didáctica de las ciencias de la naturaleza. ARI }\end{array}$ \\
\hline & La práctica profesional en la formación didáctica y pedagógica de docentes. ARN \\
\hline & $\begin{array}{l}\text { Del flogisto a la oxidación: la construcción de modelos explicativos en la formación inicial de } \\
\text { profesores de química. MEl }\end{array}$ \\
\hline \multirow{7}{*}{15} & $\begin{array}{l}\text { A Imaginação Científica como Componente do Entendimento: Subsídios para o Ensino de } \\
\text { Física. TMl }\end{array}$ \\
\hline & El modelo semicuántico de Bohr en los libros de texto. ARI \\
\hline & $\begin{array}{l}\text { Elementos de uma poética da ciência: fundamentos teóricos e implicações para o ensino de } \\
\text { ciências. TDI }\end{array}$ \\
\hline & $\mathrm{pH}$, historia de un concepto. Análisis en textos de educación superior. TMN \\
\hline & Filatelia y Didáctica de la Química: un ejemplo con los Gases Ideales. ARI \\
\hline & $\begin{array}{l}\text { La transposición didáctica de conceptos de química y su relación con la enseñanza de las } \\
\text { ciencias de la salud en el diseño del aula virtual "quisas" (química y salud, sinergias). TLI }\end{array}$ \\
\hline & $\begin{array}{l}\text { ¿Cómo se aplican los criterios de transposición didáctica en el libro de texto? Un análisis en el } \\
\text { concepto de evolución de sexto grado. MEl }\end{array}$ \\
\hline \multirow{2}{*}{17} & $\begin{array}{l}\text { Identificação de modelos no conteúdo de estrutura atômica nos livros de química geral do } \\
\text { ensino superior. MEl }\end{array}$ \\
\hline & La nueva didáctica de las ciencias naturales y los modelos científicos. ARN \\
\hline \multirow{5}{*}{18} & $\begin{array}{l}\text { Representaciones sobre la naturaleza de las ciencias y su relación con los trabajos prácticos en } \\
\text { áreas profesionalizantes de agronomía. MEI }\end{array}$ \\
\hline & $\begin{array}{l}\text { La selección, secuenciación y organización de los contenidos de biología desde el } \\
\text { Conocimiento Didáctico del Contenido (Cdc) en los profesores de secundaria en } \\
\text { Barrancabermeja. MEl }\end{array}$ \\
\hline & $\begin{array}{l}\text { Ciencia / Tecnología/ Sociedad/ Ambiente: algunos elementos a tener en cuenta en un } \\
\text { proceso de renovación de la enseñanza de las ciencias. ARN }\end{array}$ \\
\hline & $\begin{array}{l}\text { La enseñanza de las ciencias naturales en las primeras edades, su contribución a la promoción } \\
\text { de competencias de pensamiento científico. LCL }\end{array}$ \\
\hline & $\begin{array}{l}\text { El bloque académico como estructura interdisciplinaria en el programa de medicina veterinaria } \\
\text { y zootecnia en Unipaz. ARN }\end{array}$ \\
\hline \multirow{3}{*}{19} & $\begin{array}{l}\text { La necesaria renovación de la formación del profesorado para una educación científica de } \\
\text { calidad. ARN }\end{array}$ \\
\hline & CIÊNICA: divulgação da ciência e tecnologia por meio do teatro. ARI \\
\hline & La función de la tabla periódica en la enseñanza de la química. Clasificar o aprender. TDI \\
\hline \multirow{5}{*}{20} & Historia experimental de la química. MEN \\
\hline & Una vigilancia histórico y epistemológica del concepto de hibridación. MEI \\
\hline & Aproximación histórica de las comunidades académicas de ingenieros. ARN \\
\hline & El aprendizaje de la historia experimental de la Química. ARN \\
\hline & Modelos sobre las disoluciones electrolíticas en la formación inicial de profesores. ARI \\
\hline
\end{tabular}




\begin{tabular}{|c|c|}
\hline No. Art & Título y tipo del documento en el que se cita \\
\hline \multirow{4}{*}{21} & La modelización del enlace químico en libros de texto de distintos niveles educativos. ARI \\
\hline & $\begin{array}{l}\text { Modelo didáctico analógico de enlace químico: caracterización de las formas de significar de } \\
\text { los estudiantes de educación media. TMN }\end{array}$ \\
\hline & $\begin{array}{l}\text { Estudio histórico de la ionización y la polaridad para la enseñanza del concepto de enlace } \\
\text { químico. TMN }\end{array}$ \\
\hline & Unidad didáctica para la enseñanza y aprendizaje del concepto enlace químico. TMN \\
\hline \multirow{5}{*}{22} & $\begin{array}{l}\text { Modelos mentales sobre la enseñanza de docentes de la Institución Educativa Fe y Alegría la } \\
\text { Paz, del municipio de Manizales. TMN }\end{array}$ \\
\hline & $\begin{array}{l}\text { Las analogías como recurso didáctico: uso del lenguaje científico y aprendizaje de la } \\
\text { combustión. TMN }\end{array}$ \\
\hline & A qué epistemología recurrir para investigar sobre la enseñanza de las ciencias. ARN \\
\hline & Modelos mentales sobre enseñanza que poseen los docentes de educación inicial. TMN \\
\hline & $\begin{array}{l}\text { Formación de profesores de química a partir de la explicación de fenómenos cotidianos: una } \\
\text { propuesta con resultados. ARI }\end{array}$ \\
\hline 25 & Enseñanza de la cinética química por medio de simulaciones y aprendizaje activo. TMN \\
\hline \multirow[b]{2}{*}{26} & La dimensión investigativa en la formación inicial de profesores de química. LCL \\
\hline & $\begin{array}{l}\text { Institucionalización de la formación de licenciados en química en Colombia: } 1970 \text { - } 2017 \text {. El } \\
\text { caso de la Universidad Pedagógica Nacional. TDN }\end{array}$ \\
\hline
\end{tabular}

Fuente: elaboración propia.

Como se mostró en la tabla 6, y a partir de la premisa cienciométrica de que el indicador de citaciones es determinante en la evaluación cuantitativa de la producción científica, se establece con claridad cuál ha sido el impacto de la literatura puesta en circulación. Es posible afirmar que los 26 manuscritos publicados por integrantes del Grupo IREC en la revista TED, revisten una especial atención en 57 producciones intelectuales que registran citaciones de ellos. Son las tesis de maestría a nivel nacional (15), los artículos publicados en revistas tanto internacionales (12), como nacionales (11), y las memorias de eventos internacionales (7), los 5 artículos con mayor citación son los subrayados en la tabla 2.

Dicho análisis se reafirma si se tiene en cuenta que en el año 2017 los dos profesores investigadores con mayor número de publicaciones en el Grupo IREC hicieron parte de un prestigioso ranking que ponderó a los científicos colombianos con mayor índice de producción intelectual, y uno de los criterios con mayor peso en este tipo de clasificaciones es el número de citaciones hechas por otros intelectuales en sus publicaciones.

\section{Conclusiones}

Una mirada hacia atrás, de conformidad con esta investigación documental y adoptando perspectivas metodológicas de la evaluación cuantitativa de la producción científica, permite afirmar con satisfacción, que los propósitos que dieron sentido a la creación de la revista Tecné, Episteme y Didaxis - TED, así como del Congreso Internacional sobre Formación de Profesores de Ciencias, se han 
venido cumpliendo de manera paulatina, con lo cual contribuyen de modo significativo a la configuración y consolidación de colectivos de investigación en el campo de la didáctica de las ciencias experimentales, entre ellos, el Grupo de investigación Representaciones y Conceptos Científicos - IREC, cuya categorización $\mathrm{A}$ en Colciencias ha sido posible, entre otros diversos productos de nuevo conocimiento, gracias a los artículos publicados en TED como vehículo de divulgación y circulación del conocimiento científico.

El Grupo de Investigación IREC pone a disposición de la comunidad de especialistas en didáctica de las ciencias experimentales el balance de producción intelectual que aquí se ha presentado. En suma, las 26 producciones académicas de integrantes de este colectivo, junto con los 57 documentos en los que estas han sido citadas, constituyen un acervo documental que bien puede ser entendido en su conjunto como un producto de nuevo conocimiento, en tanto que sistematización con la que se aporta de manera significativa en la necesaria y urgente elaboración de un estado del arte colectivo regional de la investigación en este campo.
Ha habido falencias, como en todo emprendimiento humano, pero se han sabido superar con la colaboración de todos aquellos que han participado desinteresadamente en el mismo. Ha sido un aprendizaje permanente, que ha posibilitado que la UPN y los integrantes del Grupo IREC sean reconocidos en Iberoamérica y que, por tanto, se hayan establecido lazos de amistad y de colaboración académica que continuarán fortaleciéndose. De igual manera, se ha tenido el cuidado de formar a nuevos especialistas, los que seguirán trabajando desde perspectivas conceptuales y metodológicas innovadoras.

Es necesario resaltar que TED no solo contribuyó a consolidar el Grupo de Investigación IREC, sino que desde sus inicios lo ha hecho con otros Grupos de América Latina, al mismo tiempo que se ha constituido en un medio, para que investigadores noveles se hayan dado a conocer dentro de la comunidad que se ocupa de las investigaciones en didáctica de las ciencias. Algunos de ellos se doctoraron en universidades de Chile, Brasil y de Argentina y prestan sus servicios académicos tanto en esos países como en Colombia.

\section{Referencias}

Adúriz-Bravo, A. (2000). Consideraciones acerca del estatuto epistemológico de la didáctica específica de las ciencias naturales. Revista del Instituto de Investigaciones en Ciencias de la Educación, 9 (17), 49-52.

Aliberas, J., Gutiérrez, R. e Izquierdo, M. (1989). La didáctica de las ciencias: Una empresa racional. Enseñanza de las Ciencias, 7 (3), 227-280.

Barona, J. L. (1994). Ciencia e historia. Debates y tendencias en la historiografía de la ciencia. Valencia: Guada.

Franco Moreno, R., Gallego Badillo, R., Pérez Miranda, R. (2009). Desarrollo científico en Colombia: una revisión de las revistas científicas especializadas en ciencias de la naturaleza. Tecné, Episteme y Didaxis [No. extraordinario].

Franco Moreno, R., Gallego Badillo, R., y Pérez Miranda, R. (2015). La dimensión investigativa en la formación inicial de profesores de la upN. Revista científica, 22, 129-136. 
Franco, R., Velasco, M. y Riveros, C. (2017). Los trabajos prácticos de laboratorio en la enseñanza de las ciencias: tendencias en revistas especializadas (2012-2016). Tecné, Episteme y Didaxis, 41, 37-56.

Fraser, B., Tobin, K., \& McRobie, C. (2012). (Coords). Second international handbook of science education. Dordrecht: Springer.

Fuentes, G. (2005). Un Estado del arte de las investigaciones sobre la didáctica de las ciencias experimentales financiadas por Colciencias, 1999-2003 (Tesis de Maestría), Universidad Pedagógica Nacional.

Gallego Badillo, R. (2008). Ciencias, Revistas Especializadas y Comunidades Científicas, Tecné, Episteme y Didaxis [No. extraordinario], 9-25.

Gallego Badillo, R., Gallego Torres, A. y Pérez Miranda, R. (2012). El Congreso de Karlsruhe: los inicios de una comunidad científica. Educación Química, 23 (2), 280-283.

Gallego Badillo, R., Pérez Miranda, R. y Franco Moreno, R. (2016). (Coords). Lecturas en Didáctica de la Química. Bogotá: Universidad Pedagógica Nacional.

Gallego Badillo, R., Pérez Miranda, R., Torres de Gallego, L. y Amador Rodríguez, R. (2004). La formación inicial de profesores de ciencias en Colombia. Contrastación de fundamentos. Bogotá: Universidad Pedagógica Nacional.

Garzón, A., Zúñiga, L. y Franco, R. (2011). El campo CTSA en las revistas especializadas: 2005-2010. ¿̇Cuáles son las finalidades de la educación en ciencias desde esta línea? Tecné, Episteme y Didaxis: TED [No. extraordinario], 1443-1448.

Golombek, D. (2007). Demoliendo papers. Buenos Aires: Siglo XXI.

Gil Pérez, D. (1994). Diez años de investigación en didáctica de las ciencias: realidades y perspectivas. Enseñanza de las ciencias, 12 (2), 154-164.

Gil, D., Carrascosa, J. y Martínez-Terrades, F. (1999). El surgimiento de la didáctica de las ciencias como campo específico de conocimientos. Revista de Educación y Pedagogía, 11 (25), 13-65.

Hoffmann, R. (1997). Lo mismo y no lo mismo. México: Fondo de Cultura Económica. Kragh, H. (2007). Introducción a la Historia de la Ciencia. Barcelona: Crítica.

Kuhn, T. S. (1972). La estructura de las revoluciones científicas. México: Fondo de Cultura Económica.

Lakatos, I. (1983). La metodología de los programas de investigación científica. Madrid: Alianza.

Nardi, R. (2003). La educación en ciencias, la investigación en enseñanza de las ciencias y la formación de profesores en Brasil. Tecné, Episteme y Didaxis [No. extraordinario], 19-33. 
Pla I Brunet, J. (2003) (Coord). 10 impactos de la ciencia del siglo xx. Madrid: Fondo de Cultura Económica.

Novak, J. D. (1988). El constructivismo humano: Un consenso emergente. Enseñanza de las Ciencias, 6 (3), $213-233$.

Popper, K. (1962). La lógica de la investigación científica. Madrid: Tecnos.

Sanmartí, N. e Izquierdo, M. (2001). Cambio y conservación en la enseñanza de las ciencias ante las TIC. Alambique. Didáctica de las Ciencias Experimentales, $29,71-83$.

Soler, N. y Parga, D. (2008). ¿̇Hacia qué líneas de investigación tienden los artículos publicados en la revista TED durante los últimos 5 años? Tecné, Episteme y Didaxis: TED [No. extraordinario], 204-215.

Zambrano, A., Salazar, T., Candela, B. y Villa, L. (2017). Estado del arte de la investigación en educación en Colombia: Un caso de los programas de formación doctoral en la nación. Tecné, Episteme y Didaxis, 41, 57-71.

\section{Para citar este artículo:}

Franco, R., Gallego Badillo, R. y Pérez Miranda, R. (2019). 25 años de la revista TED: sus aportes en la consolidación de un grupo de investigación en didáctica de las ciencias experimentales. Tecné, Episteme y Didaxis:TED, 46, 169-187. 